\title{
POSSIBLE NEUTRINO-ANTINEUTRINO OSCILLATION UNDER GRAVITY AND ITS CONSEQUENCES
}

\author{
BANIBRATA MUKHOPADHYAY \\ Department of Physics, Indian Institute of Science, Bangalore-560012, India \\ bm@physics.iisc.ernet.in
}

\begin{abstract}
We show that under gravity the effective masses for neutrino and antineutrino are different which opens a possible window of neutrino-antineutrino oscillation even if the rest masses of the corresponding eigenstates are same. This is due to CPT violation and possible to demonstrate if the neutrino mass eigenstates are expressed as a combination of neutrino and antineutrino eigenstates, as of the neutral kaon system, with the plausible breaking of lepton number conservation. In early universe, in presence of various lepton number violating processes, this oscillation might lead to neutrino-antineutrino asymmetry which resulted baryogenesis from the B-L symmetry by electro-weak sphaleron processes. On the other hand, for Majorana neutrinos, this oscillation is expected to affect the inner edge of neutrino dominated accretion disks around a compact object by influencing the neutrino sphere which controls the accretion dynamics, and then the related type-II supernova evolution and the r-process nucleosynthesis.
\end{abstract}

\section{Introduction}

The neutrino oscillation, in the flat space, is due to difference in rest masses between two mass eigenstates. However, in late eighties, it was first pointed out ${ }^{1}$ that presence of gravitational field affects different neutrino flavors differently which violates equivalence principle and thus governs oscillation, even if neutrinos are massless or of degenerate mass. The neutrino oscillation with LSND data ${ }^{2}$ indeed can be explained by degenerate or massless neutrinos with flavor non-diagonal gravitational coupling. It was further $\operatorname{argued}^{3}$ that the flavor oscillation is possible in weak gravitational field with the probability phase proportional to the gravitomagnetic field. The oscillation was also shown to be feasible when the maximum velocities of different neutrino differ each other, even if they are massless. ${ }^{4}$

All the above results are for flavor oscillation or/and without considering rigorous general relativistic effects. However, properties of neutrino in curved spacetime have already been discussed ${ }^{5-7}$ in literature. Here we address the neutrino-antineutrino oscillation, which violates lepton number conservation, focusing on the nature of space-time curvature and its special effect.

While the neutrino-antineutrino oscillation under gravity is an interesting issue on its own right, the present result is able to address two long-standing mysteries in astrophysics and cosmology: (1) Source of abnormally large neutron abundance to support the r-process nucleosynthesis in astrophysical site. (2) Possible origin of baryogenesis.

\section{Oscillation probability}

Let us recall the fermion Lagrangian density in curved spacetime $e^{5,7}$

$$
\mathcal{L}=\sqrt{-g} \bar{\psi}\left[\left(i \gamma^{a} \partial_{a}-m\right)+\gamma^{a} \gamma^{5} B_{a}\right] \psi=\mathcal{L}_{f}+\mathcal{L}_{I},
$$


where

$$
B^{d}=\epsilon^{a b c d} e_{b \lambda}\left(\partial_{a} e_{c}^{\lambda}+\Gamma_{\alpha \mu}^{\lambda} e_{c}^{\alpha} e_{a}^{\mu}\right), \quad e_{a}^{\alpha} e_{b}^{\beta} \eta^{a b}=g^{\alpha \beta},
$$

the choice of unit is $c=\hbar=k_{B}=1$. $\mathcal{L}_{I}$ may be a CPT violating interaction and thus the corresponding dispersion energy ${ }^{7}$ for neutrino and antineutrino in standard model

$$
E_{\nu}=\sqrt{(\vec{p}-\vec{B})^{2}+m^{2}}+B_{0}, \quad E_{\bar{\nu}}=\sqrt{(\vec{p}+\vec{B})^{2}+m^{2}}-B_{0} .
$$

Eq. (3) tells us that under gravity neutrino energy is split up from antineutrino energy. The CPT status of $\mathcal{L}_{I}$ has been discussed in detail in our previous works. ${ }^{7}$

Now motivated by the neutral kaon system, we consider two distinct orthonormal eigenstates $\left|E_{\nu}\right\rangle$ and $\left|E_{\bar{\nu}}\right\rangle$ for a neutrino and an antineutrino type respectively. Further we introduce a set of neutrino mass eigenstates at $t=0 \mathrm{as}^{8}$

$$
\left|m_{1}>=\cos \theta\right| E_{\nu}>+\sin \theta\left|E_{\bar{\nu}}>, \quad\right| m_{2}>=-\sin \theta\left|E_{\nu}>+\cos \theta\right| E_{\bar{\nu}}>.
$$

Therefore, in presence of gravity, the oscillation probability for $\mid m_{1}(t)>$ at $t=0$ to $\mid m_{2}(t)>$ at a later time $t=t_{f}$ can be found as

$$
P_{12}=\sin ^{2} 2 \theta \sin ^{2} \delta, \quad \delta=\frac{\left(E_{\nu}-E_{\bar{\nu}}\right) t_{f}}{2}=\left[\left(B_{0}-|\vec{B}|\right)+\frac{\Delta m^{2}}{2|\vec{p}|}\right] t_{f},
$$

where we consider ultra-relativistic neutrinos. Normally, the rest mass difference of particle and antiparticle is zero and thus possible $\delta \neq 0$ is mostly due to $B_{a} \neq 0$ i.e. due to gravitational coupling. Therefore, the neutrino-antineutrino oscillation may be possible in presence of gravity provided there is a lepton number violating process. If neutrinos exhibit Majorana mass, then lepton number violation is automatically taken care. Hence, the CPT violating nature of background curvature coupling generates effective mass difference, while lepton number violating process leads to oscillation between neutrino and antineutrino.

The oscillation probability is maximum at $\theta=\pi / 4$ and is zero at $\theta=0, \pi / 2$. From eqn. (5), the oscillation length, $L_{o s c}$, by appropriately setting dimensions, is obtained as

$$
L_{o s c}=c t_{f}=\frac{\pi \hbar c}{\tilde{B}} \sim \frac{6.3 \times 10^{-19} \mathrm{GeV}}{\tilde{B}} \mathrm{~km},
$$

where $\tilde{B}=B_{0}-|\vec{B}|$ is expressed in $\mathrm{GeV}$ unit and the neutrino is considered to be moving in the speed of light.

\section{Consequence and Discussion}

One of the situations where the gravity induced neutrino-antineutrino oscillation may occur is the GUT era of anisotropic phase of early universe when $\tilde{B} \sim 10^{5} \mathrm{GeV} .^{7}$ From eqn. (6), this leads to $L_{o s c} \sim 10^{-24} \mathrm{~km}$ which is $10^{14}$ orders of magnitude larger than the Planck length. This has an important implication as the size of universe at the GUT era is within $\sim 10^{26}$ times of the Planck. Therefore, the oscillation may 
lead to leptogenesis and then to baryogenesis by electro-weak sphaleron processes due to $B-L$ conservation, what we see today.

Another plausible region for an oscillation of this kind to occur is the inner accretion disk of the neutrino dominated accretion flow (NDAF) ${ }^{9}$ around a rotating compact object which can be extended upto several thousand Schwarzschild radius. From eqns. (2) and (6) we can obtain

$$
B^{0}=-\frac{4 a \sqrt{M} z}{\bar{\rho}^{2} \sqrt{2 r^{3}}}, \quad L_{o s c} \sim \frac{1.8 x^{7 / 2} M_{s}}{a H} \mathrm{~km}=\frac{1.2 x^{7 / 2}}{a H} M,
$$

for the Kerr geometry, where $\bar{\rho}^{2}=2 r^{2}+a^{2}-x^{2}-y^{2}-z^{2}$. The detailed calculation and discussion are presented elsewhere. ${ }^{10}$ Here we choose the mass of the compact object $M=M_{s} M_{\odot}$, radius and height of the disk orbit where oscillation takes place respectively $r \sim \bar{\rho}=x M$ and $z=H M$, and we assure $\tilde{B} \sim B_{0}$. Any oscillation at the inner edge of NDAF is expected to be influenced by gravity what affects the accretion dynamics and outflow. From eqn. (7), $L_{\text {osc }}$ varies from a few factors to several hundreds of Schwarzschild radii at $x \leq 10$ for a fast spinning compact object.

Supernova is thought to be the astrophysical site of the r-process nucleosynthesis. During supernova, neutron capture processes for radioactive elements take place in presence of abnormally large neutron flux. However, how does the large neutron flux arise is still an open question. There are two related reactions:

$$
n+\nu_{e} \rightarrow p+e^{-}, \quad p+\bar{\nu}_{e} \rightarrow n+e^{+} .
$$

If $\bar{\nu}_{e}$ is over abundant than $\nu_{e}$, then, from eqn. (8), neutron production is expected to be more than proton production into the system. Therefore, the possible conversion of $\nu_{e}$ to $\bar{\nu}_{e}$ due to the gravity induced oscillation explains the overabundance of neutron.

\section{References}

1. M. Gasperini, Phys. Rev. D 38, 2635 (1988).

2. R. B. Mann, \& U. Sarkar, Phys. Rev. Lett. 76, 865 (1996).

3. D. V. Ahluwalia, \& C. Burgard, Gen. Rel. Grav. 28, 1161 (1996); Phys. Rev. D57, 4724 (1998)

4. S. R. Coleman, \& S. L. Glashow, Phys. Lett. B405, 249 (1999).

5. J. Schwinger, Particles, Sources and Fields, (Addison-Wesley, 1970).

6. J. F. Nieves, \& P. B. Pal, Phys.Rev. D58, 096005 (1998).

7. P. Singh, \& B. Mukhopadhyay, Mod. Phys. Lett. A18, 779 (2003); B. Mukhopadhyay, Mod. Phys. Lett. A20, 2145 (2005); U. Debnath, B. Mukhopadhyay, \& N. Dadhich, Mod. Phys. Lett. A21, 399 (2006).

8. G. Barenboim, J. F. Beacom, L. Borissov, \& B. Kayser, Phys. Lett. B537, 227 (2002).

9. R. Narayan, T. Piran, \& P. Kumar, Astrophys. J. 557, 949 (2001); K. Kohri, R. Narayan, \& T. Piran, Astrophys. J. 629, 341 (2005).

10. B. Mukhopadhyay, Class. Quantum Grav. 24, 1433 (2007). 